\section{LSE- and LSM-Mode Sheet Impedances of Thin Conductors}

Smain Amari and Jens Bornemann

\begin{abstract}
The sheet resistance of very thin conductors is commonly taken as $R=1 / \sigma t$. We show that the sheet impedance, defined as the ratio of the tangential electric field at the surface of the conductor to the conduction current per unit length in the conductor, depends on the field distribution. The LSE (TE-to-y) and LSM (TM-to-y) modes used in the spectral domain immittance approach have sheet impedances which are distinct for vanishingly small or large values of the wavenumber $\gamma$ in the medium surrounding a thin conductor. In the limit $\gamma \rightarrow 0$ and $t / \delta \ll 1, Z_{s h}^{\mathrm{LSE}}$ approaches $R=1 / \sigma t$ while $Z_{s h}^{\mathrm{LSM}} \rightarrow 2 / \sigma t$. In the limit $\gamma \rightarrow \infty$ and $t / \delta \ll 1, Z_{s h}^{\mathrm{LSE}}$ approaches $\stackrel{s h}{R}=2 / \sigma t$ and $Z_{s h}^{\mathrm{LSM}}$ approaches $R=1 / \sigma t$. When $t / \delta \gg 1$, the sheet impedance approaches the surface impedance $Z_{s}=(1+j) / \sigma \delta$ and is independent of the field distribution.
\end{abstract}

\section{INTRODUCTION}

Recently there has been a rising interest in the concept of surface impedance of thin conducting layers. This concept has been used to account for metallic losses in planar circuits at microwave frequencies where the skin effect plays a major role. The convenience of the approach using the surface impedance resides in the simplicity in including the boundary conditions [1]-[5]. In fact, it allows one to avoid solving the problem in numerous subregions, which can be numerically intensive. The method has, however, its shortcomings. In addition to being approximate, a problem arises when the thickness of the conducting strip is much smaller than the skin depth in the material. The situation where the thickness is much larger ( 4 times, say) than the skin depth is well handled using a surface impedance given by

$$
Z_{s}=\frac{1+j}{\sigma \delta}
$$

where $\sigma$ is the bulk conductivity of the material and $\delta$ the skin depth

$$
\delta=\sqrt{\frac{2}{\omega \sigma \mu}} .
$$

As the thickness $t$ of the conducting layer is decreased, $Z_{s}$ is replaced by the sheet resistance $R$, which reflects the fact that the cross section is the dominant property, hence leading to the singular dependence [1]

$$
R=\frac{1}{\sigma t} \text {. }
$$

However, this expression neglects the dependence of the sheet resistance on the field distribution and the remaining characteristics of the structure.

Therefore, in this paper, we propose to show that the sheet impedance, which we define as the ratio of the tangential component of the electric field at the surface of the conductor to the conduction current per unit length, namely

$$
Z=\frac{E(y=0)}{J}
$$

(c.f., Fig. 1) depends on the field distribution. The LSE- and LSMmode sheet impedances depend on the spatial variation, in the

Manuscript received June 11. 1995: revised February 15, 1996.

The authors are with the Center for Advanced Materials and Related Technology, University of Victoria, Victoria BC, V8W 3P6 Canada.

Publisher Item Identifier S 0018-9480(96)03791-X.

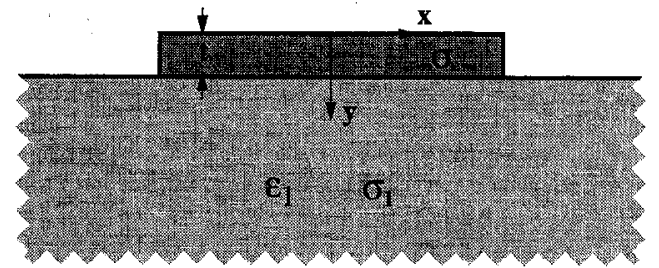

Fig. 1. A conducting strip on top of an infinite dielectric substrate.

direction normal to the conductor, of the fields in the medium under the conductor. When the field in the medium is changing either too slowly or too fast, as a function of $y$, the sheet impedances of the two modes have the ratio of two in the limit $t \rightarrow 0$. For thick conductors, the sheet impedance is independent of the field distribution and the medium, as it approaches the usual surface impedance given by (1). The analysis focuses on the LSE and LSM modes because of their importance in the spectral domain immittance approach.

Since the surface impedance $Z_{s}$, which is usually defined as the ratio of the tangential electric and magnetic fields of the conductor, also shows a dependence on the field distribution and the medium surrounding the conductor, especially for thin conductors, we adopted the term sheet impedance $Z_{s h}$ in this paper to distinguish between the two quantities which coincide only for thick conductors. In order to clarify the difference between the two terms, consider a conductor of thickness $t$ and conductivity $\sigma$ on top of a thick conductor of conductivity $\sigma_{1}$. According to $\left[6\right.$, p. 300], the surface impedance $Z_{s}$ is found as

$$
\frac{Z_{s}}{R_{s}}=(1+j) \frac{\sinh (t \tau)+\frac{R_{s 1}}{R_{s} \cosh (t \tau)}}{\cosh (t \tau)+\frac{R_{s 1}}{R_{s} \sinh (t \tau)}}
$$

where $R_{s}$ and $R_{s 1}$ are given by

$$
\begin{aligned}
R_{s} & =\frac{1}{\sigma \delta}, \\
R_{s 1} & =\frac{1}{\sigma_{1} \delta_{1}} \\
\tau & =\frac{1+j}{\delta}
\end{aligned}
$$

and $\delta$ is the penetration depth. If, in this expression, one lets the conductivity $\sigma_{1}$ approach zero (as in the case of a conductor on a substrate) such that the conduction current is nonzero only in the thin conductor, one gets $[7$, p. 128], $[8$, p. 154]

$$
Z_{s h}=(1+j) R_{s} \operatorname{coth}(t \tau) .
$$

The notation $Z_{s h}$ is used to emphasize the fact that it is equal to the ratio of the tangential electric field to the conduction current per unit length in the conductor. From this last equation it can be seen that, in the limit $t / \delta \ll 1$, the sheet impedance indeed depends on $t$ according to (3).

It is also worth emphasizing that the derivation of (4) assumes no spatial variation of the fields in the plane parallel to the conducting sheet. We will show that our expressions for the sheet impedance reduce to (7) under these conditions. In [8, p. 154] it is mentioned that (7) is a good approximation as long as the wave impedance of the medium under the conductor is much larger that the intrinsic impedance of the conductor. The conditions under which this approximation holds are examined in this paper. 


\section{SHEET IMPEDANCE OF A CONDUCTING STRIP}

The structure under consideration is shown in Fig. 1. It consists of a conducting strup of thickness $t$. which we assume much smaller than its width $w$, and conductivity $\sigma$ on top of an ideal $\left(\sigma_{1}=0\right)$ dielectric substrate of permittivity $\epsilon_{\mathbf{1}}$. The substrate is assumed infinitely thick, although this is not necessary. The finite thickness determines the wave impedance at the conductor-dielectric interface and does not affect the conclusions of the analysis.

In the spectral domain approach, the tangential electric field at the conductor-dielectric interface is related to the current density through the Green's impedance dyadics [9], [10]. In applying the method of moments (Galerkin method), it is necessary to establish an additional relationship, in the metallized region, between the current in the conductor and the tangential electric field [11]. Such a relationship should reflect Ohm's law and states that the current is, indeed, a conduction current and is related to the electric field by

$$
\boldsymbol{J}=\sigma \boldsymbol{E}
$$

Since the field distribution in this and similar multilayered structures can be decomposed into LSE and LSM modes, it is sufficient to analyze each one separately since they are decoupled [9]. [10]. The field components are Fourier transformed in the $x z$ plane. Let $\alpha$ and $\beta$ denote the arguments of the Fourier transforms. The $y$-dependence is then of the form

$$
\frac{d^{2} \phi}{d y^{2}}=\gamma^{2} \phi
$$

where $\phi$ is a generic field component, $\gamma^{2}=\alpha^{2}+\beta^{2}-k_{\imath}^{2}$ and $k_{2}^{2}=\omega^{2} \epsilon_{\imath} \mu_{0} \epsilon_{0}$.

It is convenient to carry out the analysis in a system of axes which is rotated in the $x z$ plane by an angle $\theta$ given by

$$
\theta=\tan ^{-1}\left(\frac{\alpha}{\beta}\right)
$$

as shown in Fig. 2. In the new system of axes $(\boldsymbol{u}, \boldsymbol{v}, \boldsymbol{y})$, the LSE mode has components $\left(H_{y}, E_{u}, H_{v}\right)$ and is generated only by the current in the u drection. Similarly, the LSM mode has components $\left(E_{y}, H_{u}, E_{v}\right)$ and is generated only by the current in the $\mathbf{v}$ direction. It is this decoupling of the modes in the new system of coordinates which allows us to treat the LSE and LSM modes separately. For convenience we introduce the quantity $a$ such that

$$
a^{2}=\frac{\alpha^{2}+\beta^{2}}{k_{1}^{2}} \text {. }
$$

The electric field component $E_{u}$ in the conductor is of the form

$$
E_{u}(y)=A e^{-\gamma_{c} y}+B e^{\gamma c y} .
$$

Where $A$ and $B$ are constants to be determined from the boundary conditions and $\gamma_{c}^{2}=\alpha^{2}+\beta^{2}+j \omega \mu_{0}\left(\sigma+j \omega \epsilon_{0}\right)$. For a good conductor, $\gamma_{c}$ can be approximated by

$$
\begin{aligned}
\gamma_{c} & \simeq(1+j) \sqrt{\frac{\sigma \mu_{0} \omega}{2}} \\
& =\frac{1+j}{\delta} .
\end{aligned}
$$

The magnetic field component $H_{v}$ then follows from Maxwell's equations

$$
H_{v}(y)=\frac{1}{z_{c}}\left(A e^{-\gamma c y}-B e^{\gamma c y}\right)
$$

where $z_{c}$ is the intrinsic impedance of the conductor. $z_{c}=(1+j) / \sigma \delta$.

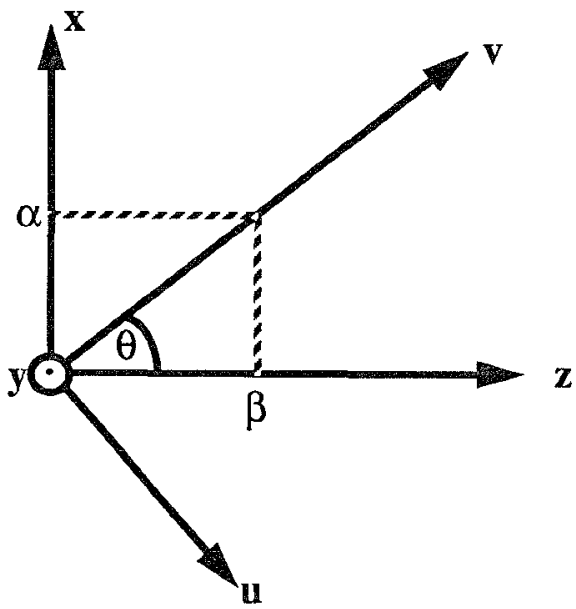

FIg. 2. Axes transformation to decouple the LSE and LSM modes.

In the medium under the conductor, the field component can similarly be expressed as

$$
E_{u}(y)=C e^{-\gamma 1 y}
$$

and

$$
H_{v}(y)=Y_{1}^{-\mathrm{LSE}} C e^{-\gamma_{1} y}
$$

where $Y_{1}^{\mathrm{LSE}}=\gamma_{1} / j \omega \mu_{0}$. The current per unit length in the conducting strip is equal to the integral of $\sigma E_{u}(y)$ over the thickness of the strip or

$$
J_{u}=\frac{\sigma}{\gamma_{c}}\left[A\left(1-e^{-\gamma_{c} t}\right)+B\left(e^{\gamma_{c} t}-1\right)\right] .
$$

The sheet impedance is then given by

$$
\begin{aligned}
Z_{s h}^{\mathrm{LSE}} & =\frac{E_{u}(y=0)}{J_{u}} \\
& =\frac{\gamma_{c}}{\sigma} \frac{\frac{A}{B}+1}{\frac{A}{B}\left(1-\epsilon^{-\gamma_{c} t}\right)+\left(e^{\gamma^{t} t}-1\right)} .
\end{aligned}
$$

Requiring the continuty of the tangential electric and magnetic field components at the interface $y=t$ gives the ratio $A / B$

$$
\frac{A}{B}=e^{2 \gamma_{c} t} \frac{1+Y_{1}^{\mathrm{LSE}} z_{c}}{1-Y_{1}^{\mathrm{LSE}} z_{c}} .
$$

Combining (18) and (19) we get the final expression of the sheet impedance of the LSE mode

$$
\begin{aligned}
& Z_{s h}^{\mathrm{LSE}}\left(a, \gamma_{c}, t\right)= \\
& \frac{1+j}{\sigma \delta} \frac{1+Y_{1}^{\mathrm{LSE}} z_{c} \tanh \left(\gamma_{c} t\right)}{\tanh \left(\gamma_{c} t\right)+\frac{\cosh \left(\gamma_{c} t\right)-1}{\cosh \left(\gamma_{c} t\right)} \mathbf{Y}_{1}^{\mathrm{LSE}}{\tilde{\sim}_{c}}} .
\end{aligned}
$$

The expression for $Z_{s h}^{\mathrm{LSM}}$ is obtained similarly and is given by

$$
\begin{aligned}
& Z_{s h}^{\mathrm{LSMI}}\left(a, \gamma_{c}, t\right)= \\
& \frac{1+j}{\sigma \delta} \frac{1+\mathrm{I}_{1}^{-\mathrm{LSM}} z_{c} \tanh \left(\gamma_{c} t\right)}{\tanh \left(\gamma_{c} t\right)+\frac{\cosh \left(\gamma_{c} t\right)-1}{\cosh \left(\gamma_{c} t\right)} Y_{1}^{\mathrm{LSM}} z_{c}}
\end{aligned}
$$

where $I_{1}^{\text {L.SA }}=j w \epsilon_{1} \epsilon_{0} / \gamma_{1}$.

If the substrate is of finite thickness, the sheet impedance for the two modes can be obtained by replacing $Y_{1}$ by the appropriate wave impedance seen from under the conductor in the positive $y$-direction (Fig. 1). 


\section{RESUltS AND DisCussion}

As mentioned in the introduction, [8, p. 154] states that (7) gives a good approximation to the sheet impedance when the wave impedance $1 / Y_{1}$ is much larger than the intrinsic impedance of the conductor $z_{c}$. That (20) and (21) both reduce to (7) can be seen straightforwardly by taking $Y_{1}^{r} z_{c} \rightarrow 0$.

Both (20) and (21) give a sheet impedance which approaches the usual surface impedance in the limit of a thick conductor regardless of the value of $Y_{1}$. A thick enough conductor attenuates the incident wave such that no reflections between its surfaces take place.

Of more importance and interest, however, is the dependence of the sheet impedance on the thickness of thin conductors. In the limit $t \rightarrow 0$, the sheet impedance approaches the sheet resistance $R=1 / \sigma t$ as long as the quantity $Y_{1} z_{c}\left(Y_{1}\right)$ is not large. We now examine the conditions under which $Y_{1}$ is large.

For the LSM mode, $\mathrm{I}_{1}^{-\mathrm{LSM}}=j \omega \epsilon_{1} \epsilon_{0} / \gamma_{1}$. Clearly, this quantity is large when $\gamma_{1}$ approaches zero. Under the same conditions, $Y_{1}^{\text {LSE }}$ approaches zero. By expanding the hyperbolic functions in Taylor series, (20) and (21) lead to the following interesting relations

$$
Z_{s h}^{\mathrm{LSM}} \rightarrow \frac{2}{\sigma t}, \quad \gamma_{1} \rightarrow 0, \quad t \rightarrow 0
$$

and

$$
Z_{s h}^{\mathrm{LSE}} \rightarrow \frac{1}{\sigma t}, \quad \gamma_{1} \rightarrow 0, \quad t \rightarrow 0 .
$$

In other words, the LSM modes have a sheet resistance equal to twice that of the LSE modes when the conductor's thickness is much smaller than the penetration depth and $\gamma_{1}$ approaches zero.

The order is reversed when $\gamma_{1}$ is large where $Y_{1}^{\mathrm{LSM}}$ approaches zero and $Y_{1}^{\mathrm{LSE}}$ is large. In this case the following relations hold

and

$$
Z_{s h}^{\mathrm{LSM}} \rightarrow \frac{1}{\sigma t}, \quad \gamma_{1} \rightarrow \infty, \quad t \rightarrow 0
$$

$$
Z_{s h}^{\mathrm{LSE}} \rightarrow \frac{2}{\sigma t}, \quad \gamma_{1} \rightarrow \infty, \quad t \rightarrow 0 .
$$

Now it is the LSE sheet impedance which is twice the sheet resistance.

When the thickness is comparable to the penetration depth, the sheet impedance of the two sets of modes are still different. In order to compare the sheet impedances, as given by (20) and (21), to both the sheet resistance $R=1 / \sigma t$ and the surface resistance $R_{s}=1 / \sigma \delta$, we plot the ratios $Z_{s h} / R$ and $Z_{s h} / R_{s}$ as a function of $t / \delta$.

Fig. 3 shows the real and imaginary parts of these ratios for a low value (unity) of the parameter $a$ defined in (11). Although the case $a=1$ is a particular case, the following results hold for values of $a$ which are close enough to unity such that $\gamma_{1}$ approaches zero. The conductor is assumed copper with $\sigma=5.88\left(10^{7}\right) \mathrm{S} / \mathrm{m}$. It is clearly seen that the real part of the LSE-mode sheet impedance normalized to $R_{s}$ approaches unity for a thick conductor and is much larger than unity for small values of $t / \delta$. The same behavior is observed for the ratio of the real part of $Z_{s h}^{\mathrm{LSM}}$ to $R_{s}$, and the factor of two between $Z_{s h}^{\mathrm{LSM}}$ and $Z_{s h}^{\mathrm{LSE}}$, in the region of small values of $t / \delta$, is obvious. The corresponding imaginary parts are plotted in solid lines. They vanish in the limit of thin conductors and approach unity for $t / \delta$ larger than about 2. However, the ratio of the imaginary part of $Z_{s h}^{\mathrm{LSM}}$ to $R_{s}$ approaches unity faster than that of $Z_{s h}^{\mathrm{LSE}}$ to $R_{s}$. It is interesting to note that the inductance of the LSM mode is consistently larger than that of the LSE mode except for thick or very thin conductors. The dotted lines represent the ratio of the real part of $Z_{s h}^{\mathrm{LSE}}$ and $Z_{s h}^{\mathrm{LSM}}$ to the sheet resistance $R$. For values of $t / \delta$ smaller than about 1 , these ratios are practically constant. Again, note the factor of two between these curves for small values of $t / \delta$. The ratio of the real part of $Z_{s h}^{\mathrm{LSM}}$ to $R$ equals two times that of $Z_{s h}^{\mathrm{LSE}}$ to $R$.

Fig. 4 shows the same ratios for a large value of $a\left(a=10^{6}\right)$. Although the plotted curves look similar to those in Fig. 3, note

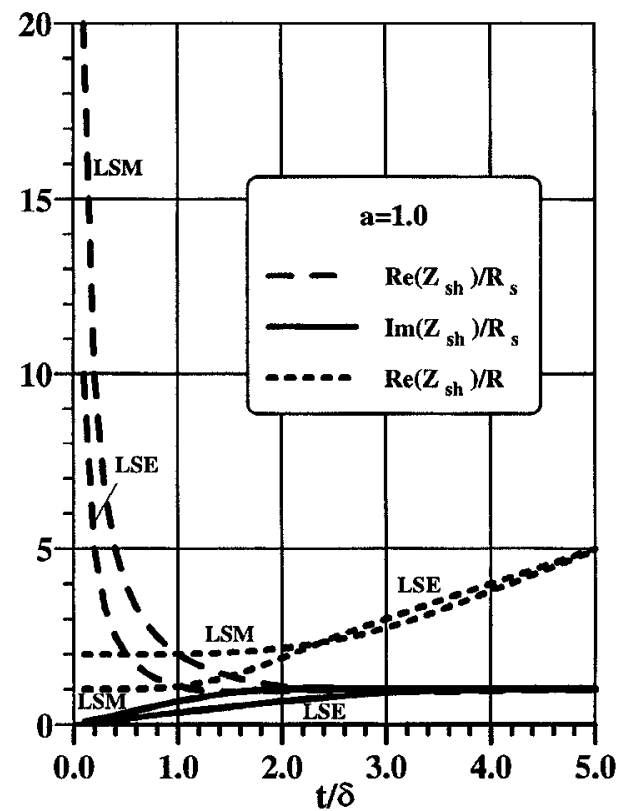

Fig. 3. Ratios of the real and imaginary parts of the sheet impedances to the real part of the surface impedance of thick conductors $R_{s}=1 / \sigma \delta$ and sheet resistance $R=1 / \sigma t$ as a function of $t / \delta$ for the LSE and LSM modes when $a=1 . \sigma=5.88\left(10^{7}\right) \mathrm{S} / \mathrm{m}$ and $\mathrm{F}=10 \mathrm{GHz}$.

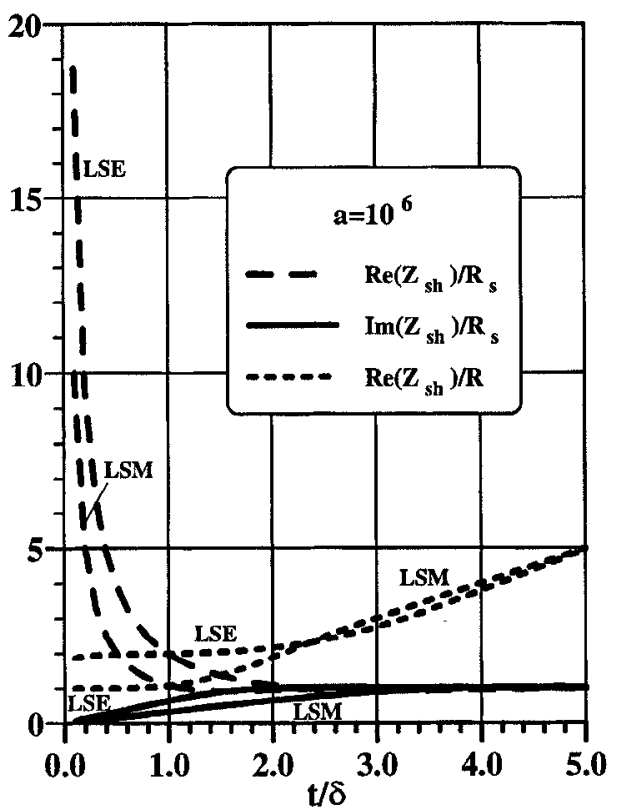

Fig. 4. Ratios of the real and imaginary parts of the sheet impedances to the real part of the surface impedance of thick conductors $R_{s}=1 / \sigma \delta$ and sheet resistance $R=1 / \sigma t$ as a function of $t / \delta$ for the LSE and LSM modes when $a=10^{6} . \sigma=5.88\left(10^{7}\right) \mathrm{S} / \mathrm{m}$ and $\mathrm{F}=10 \mathrm{GHz}$.

that it is now the LSE-mode sheet impedance that leads that of the LSM mode by a factor of two for small $t / \delta$. Moreover, the sheet inductance of the LSE mode is now consistently larger than that of the LSM mode as evidenced by the solid lines in Fig. 4. Therefore, both Figs. 3 and 4 confirm the asymptotic behavior of the LSE- and LSM-mode sheet impedances given in (22)-(25). Large value of $\gamma_{1}$ become more significant for tightly coupled and narrow lines where the current profiles change rapidly in space. Their Fourier components contain long tails which are significant at large values of $\alpha$ and $\beta$. 


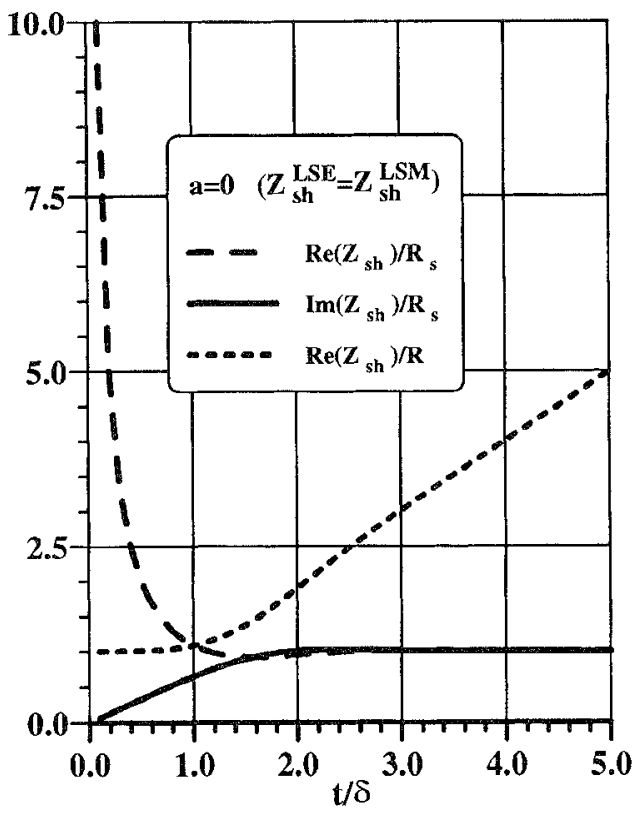

Fig. 5. Ratios of the real and imaginary parts of the sheet impedances to the real part of the surface impedance of thick conductors $R_{s}=1 / \sigma \delta$ and sheet resistance $R=1 / \sigma t$ as a function of $t / \delta$ for the LSE and LSM modes when $a=0 . \sigma=5.88\left(10^{\top}\right) \mathrm{S} / \mathrm{m}$ and $\mathrm{F}=10 \mathrm{GHz}$.

It is also interesting to note that the sheet resistance and surface impedance are usually derived for a field distribution which depends only on the coordinate normal to the plane, i.e., only on $y$. In the present analysis, this corresponds to the specific case where $a=0$. Indeed, only under these conditions, the two sheet impedances of the two modes are equal for all values of the thickness as shown in Fig. 5.

\section{CONCLUSION}

This paper presents a detailed study of the concept of sheet impedance, defined as the ratio of the tangential electric field at the surface of a conductor to the conduction current per unit length it carries. We report that the sheet resistance depends on the field distribution in the structure. In the limit of thin conductors, the sheet impedance of a LSM mode is twice that of a LSE mode when the field varies slowly in the direction normal to the conductor $(a \rightarrow 1)$. When the fields vary rapidly in the same direction, the sheet impedance of a LSE mode is twice that of a LSM mode when the conductor is very thin $(t / \delta \ll 1)$. In the limit of thick conductors the sheet impedance approaches $(1+j) / \sigma \delta$ and is independent of the field distribution.

\section{REFERENCES}

[1] I M. Pond, C. M. Krowne, and W C. Carter, "On the application of complex resistive boundary conditions to model transmission lines consisting of very thin superconductors," IEEE Trans. Microwave Theory Tech., vol. 37. no 1. pp 181-190, Jan. 1989.

[2] C. W. Kuo and T. Itoh. "A flexible approach combining the spectral domain method and impedance boundary condtion for the analysis of microstrip line," IEEE Microwave and Guided Wave Lett., vol. 1, pp. 172-174, July 1991

[3] A. T. Shalaby, E. M. Zieur, and A. O. Attıa, "Spectral domain analysis of high- $T_{c}$ superconducting microstrip resonators," in IEEE AP-S Int. Symp. Dig. 1993. pp. 193-196
[4] J C. Liou and K. M Lau, "A solution to characteristics of planar transmission lines made of finite-thickness metal on multilayered media," in IEEE MTT-S Int Microwave Symp Dig, 1990, pp. 179-181.

[5] Z Cai and J Bomemann, "Generalized spectral-domain analysis for multilayered complex media and high- $T_{C}$ superconductor applications," IEEE Trans. Microwave Theory Tech, vol. 40. pp 2251-2257, Dec. 1992.

[6] S Ramo, J. R. Whinnery, and T. Van Duzer. Fields and Waves in Communicaton Electronics. New York. W1ley, 1965, ch. 5.

[7] R. E. Matick. Transmission Lines for Digital and Communtcation Networks. New York: McGraw-Hill, 1969.

[8] E. C. Jordan and K. G. Balmain. Electromagnetıc Waves and Radiatmg Systems. Englewood Cliffs: Prentice-Hall, 1968, pp. 153-158.

[9] D. Murshekar-Syahkal, Spectral Domain Method for Microwave Integrated Circuts. New York: Wiley, 1990.

[10] T. Itoh, "Spectral domain immittance approach for dispersion characteristics of generalized printed transmission lines," IEEE Trans. Microwaie Theory Tech., vol. MTT-28, pp. 733-736, July 1980.

[11] C. M. Krowne, "Relationships for Green's function spectral dyadics unvolving anisotropic umperfect conductors imbedded in layered anisotropic media," IEEE Anternas Propagat., vol. 37, pp. 1207-1211, Sept. 1989.

\section{Accurate Analysis of Losses in Waveguide Structures by Compact Two-Dimensional FDTD Method Combined with Autoregressive Signal Analysis}

Masafumi Fujii and Sumio Kobayashi

Abstract-An efficient two-dimensional finite-difference time-domain (2-D FDTD) method combined with an autoregressive (AR) signal analysis has been proposed for analyzing the propagation properties of microwave guiding structures. The method is especially suitable for analyzing lossy transmission lines; and in contrast with previous approaches, it is based on an algorithm of a real domain only. The algorithm is verified by comparing the numerical results with exact solutions for dielectric loaded rectangular waveguides. The conductor losses in a variety of microstrip lines and coplanar waveguides have been accurately estimated by solving the electromagnetic fields in the conductors directly.

\section{INTRODUCTION}

This paper proposes a new algorithm based on two-dimensional finite-difference time-domain (2-D FDTD) method [1]-[3] combined with an autoregressive (AR) signal analysis [4] for predicting the conductor losses in microwave circuits such as in monolithic microwave/millimeter-wave integrated circuits (MMIC's) and multichip modules (MCM's). In previous 2-D FDTD methods [1], the wavegurde structures are assumed to be uniform and infinitely long in the direction of wave propagation (say z), and support modes with propagation constants $\beta$ independent of $z$. Those mean that the $z$ derivative can be replaced with $i \beta$, and result in a formulation of the algorithm in complex domain. Recently new algorithm have been proposed for enabling 2-D FDTD analysis in real domain [2], [3], however, those are restricted in the analysis of loss-less lines

In contrast with previous approaches, we assume that the waveguide has a finite length $l$, and is bounded with two infinitely large

Manuscript rece1ved August 31, 1995; rev1sed February 15, 1996.

The authors are with the Sumitomo Metal Industries. Ltd., 1-8 Fuso-cho, Amagasakı, 660 Japan.

Publisher Item Identifier S 0018-9480(96)03793-3. 